\title{
COVID-19 vaccines and the role of other potential allergenic components different from PEG.
}

\author{
Natalija Novak ${ }^{1}$, Cezmi Akdis ${ }^{2}$, and Beatriz Cabanillas ${ }^{1}$ \\ ${ }^{1}$ University of Bonn \\ ${ }^{2}$ University of Zurich
}

January 25, 2021

COVID-19 vaccines and the role of other potential allergenic components different from PEG. A reply to: "Other excipients than PEG might cause serious hypersensitivity reactions in COVID-19 vaccines"

\section{To the Editor:}

Thank you for the correspondence of Borgsteede et al "Other excipients than PEG might cause serious hypersensitivity reactions in COVID-19 vaccines". ${ }^{1}$

We agree with the authors and their observations indicating that other components different from polyethylene glycol (PEG) in the vaccines currently available for the prevention of COVID-19 could also have a potential role in hypersensitivity reactions described. In our editorial, we focused on BNT162b2 vaccine developed by BioNTech and Pfizer given special attention to the excipient PEG $2000 \mathrm{~g} / \mathrm{mol}$ (PEG-2000) contained in the vaccine in form of a PEGylated micellar carrier as a potential culprit of the reported anaphylactic reactions. PEG-2000 is among the excipients from BNT162b2 the most likely to produce an allergic response. As we focused on the first developed COVID-19 vaccine, our first editorial did not cover other vaccines developed for COVID-19. In that regard, we have recently pointed out in a follow-up article that the excipient tromethamine (also named trometamol) contained in the Moderna mRNA-1273 vaccine could contribute to the induction of anaphylactic reactions in sensitized individuals as well. ${ }^{2}$ As Borgsteede et $\mathrm{al}^{1}$ and ourselves ${ }^{2}$ indicated, a recent article has reported the first case of anaphylaxis to trometamol as an ingredient in a gadolinium-based contrast agent (GBCA). The reaction was induced shortly after the injection of GBCA and an IgE-mediated mechanism against trometamol could be detected. ${ }^{3}$ Although both BNT162b2 and mRNA-1273 vaccines are mRNA vaccines containing PEG-2000, the excipient tromethamine is exclusive from the latter (Figure 1).

The third vaccine for the prevention of COVID-19 has been recently approved in the United Kingdom. The vaccine, named AZD1222, developed by Oxford University and AstraZeneca, consists of a replication-deficient chimpanzee adenovirus (ChAdOx1) vector that contains the gene of the glycoprotein spike $(\mathrm{S})$ antigen of SARS-CoV-2. Although the AZD1222 DNA vaccine does not contain PEG-2000 or other PEGs with different molecular weights, as Borgsteede et al pointed out, ${ }^{1}$ it does contain the structurally related compound polyoxyethylene-80-sorbitan monooleate or polysorbate 80 (also named Tween 80) (Figure 1). The crossreactivity between polysorbate 80 and PEGs seems to be due to a shared moiety $-\left(\mathrm{CH}_{2} \mathrm{CH}_{2} \mathrm{O}\right)_{n}$ contained in both compounds. Polysorbate 80 is widely used as an emulsifier and stabilizer in the food industry and it is also a common excipient in medications and vaccines such as influenza, Hepatitis B, Human papillomavirus (HPV) vaccines, with the function of facilitating the vaccine components to stay soluble. Polysorbates 20, 40, and 60 also exist. Polysorbates 80 and 20 have also been included as excipients in other SARS-CoV-2 vaccines currently under evaluation in phases I, II, or III trials and that are based on adenovirus vectors or 
on the recombinant glycoprotein (S) spike antigen. ${ }^{4}$ Up to date, most of the cases of allergic reactions to polysorbates have been associated with medicines containing this excipient and only a few cases of allergy to vaccines due to polysorbates have been reported. ${ }^{5,6}$ The cases could be underrepresented due to the general unawareness among physicians of the allergenic potential of excipients such as polysorbates that may be behind an unclear allergic reaction to a given vaccine.

The role of other vaccines components such as 1,2-distearoyl-sn-glycero-3-phosphocholine (DSPC) or EDTA as potential triggers of hypersensitivity reactions should also be considered (Figure 1).

Although in general terms allergic reactions to vaccines are rare, the fact that the newly developed vaccines for the prevention of COVID-19 will be applied to a considerable majority of the world population, makes it important for health care providers to be aware of the potential threat of the excipients from COVID-19 vaccines with the potential to produce hypersensitivity reactions before the administration of the vaccines.

Beatriz Cabanillas ${ }^{1}$

Cezmi A. Akdis ${ }^{2,3}$

Natalija Novak ${ }^{4 *}$

${ }^{1}$ Department of Allergy, Research Institute Hospital Doce de Octubre, Madrid, Spain

${ }^{2}$ Swiss Institute of Allergy and Asthma Research (SIAF), University of Zurich, Davos, Switzerland.

${ }^{3}$ Christine Kühne-Center for Allergy Research and Education, Davos, Switzerland

${ }^{4}$ Department of Dermatology and Allergy, University Hospital Bonn, Bonn, Germany.

*Corresponding author: Univ.-Prof. Dr. Natalija Novak

Department of Dermatology and Allergy, University Hospital, Bonn, Venusberg Campus 1, 53127 Bonn, Germany.

Email: natalija.novak@ukbonn.de

\section{Conflict of interest}

The authors declare no conflict of interest

\section{References}

1. Borgsteede S, Geersing TH, Tempels-Pavlica Z. Other excipients than PEG might cause serious hypersensitivity reactions in COVID-19 vaccines. Allergy. 2021.

2. Ludger Klimek, Natalija Novak, Beatriz Cabanillas, et al. Allergenic components of the mRNA-1273 vaccine for COVID-19: possible involvement of polyethylene glycol and IgG-mediated complement activation. Authorea. January 01, 2021.

3. Lukawska, J., et al., Anaphylaxis to trometamol excipient in gadolinium-based contrast agents for clinical imaging. J Allergy Clin Immunol Pract, 2019. 7(3): p. 1086-1087.

4. Castells MC, Phillips EJ. Maintaining Safety with SARS-CoV-2 Vaccines. N Engl J Med. 2020 Dec 30. doi: 10.1056/NEJMra2035343.

5. Badiu I, Geuna M, Heffler E, Rolla G. Hypersensitivity reaction to human papillomavirus vaccine due to polysorbate 80. BMJ Case Rep. 2012 May 8;2012:bcr0220125797.

6. Stone CA Jr, Liu Y, Relling MV, Krantz MS, Pratt AL, Abreo A, Hemler JA, Phillips EJ. Immediate Hypersensitivity to Polyethylene Glycols and Polysorbates: More Common Than We Have Recognized. J Allergy Clin Immunol Pract. 2019;7:1533-1540.e8. 
Figure 1. Active components and excipients listed in BNT162b2, mRNA-1273, and AZD1222 vaccines. The principles of the PEGylated-lipid nanoparticles as a delivery system for the mRNA in BNT162b2, mRNA1273 vaccines are depicted in the upper part together to the replication-deficient adenovirus ChAdOx1 vector containing the gene of the glycoprotein spike (S) antigen of SARS-CoV-2 for the AZD1222 vaccine. Potential triggers of allergic reactions are indicated in red color in the excipient lists of each vaccine.
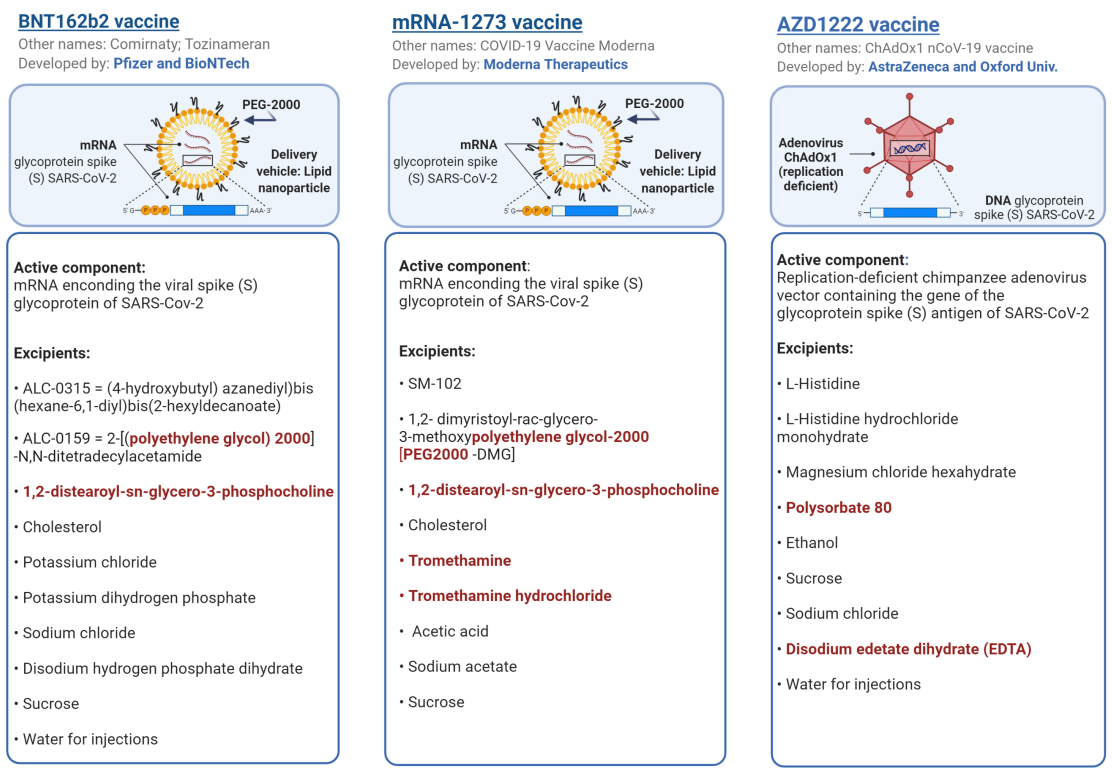